\title{
Analytical and Experimental Evaluation of Wake-up Receivers based protocols
}

\author{
Fayçal Ait Aoudia ${ }^{1}$, Michele Magno ${ }^{2}$, Matthieu Gautier ${ }^{1}$, Olivier Berder ${ }^{1}$, and Luca Benini ${ }^{2}$ \\ ${ }^{1}$ IRISA, University of Rennes 1 \\ ${ }^{2}$ ETH Zürich
}

\begin{abstract}
Achieving energy efficient wireless communication is the most pursued goal in Wireless Sensor Networks (WSNs), as energy consumption is typically a major barrier to long term applications. In recent years, ultra-low power Wake-up Receivers (WuRx) have emerged, enabling pure asynchronous wireless communication that eliminates energy waste due to idle listening. However, to achieve a significant increase of energy efficiency compared to traditional duty-cycling approaches, Medium Access Control (MAC) protocols exploiting WuRx must be carefully designed. Therefore, we propose an analytical framework to model MAC protocols, leveraging WuRx or not, which gives an important evaluation of power consumption, latency and reliability. This framework was used to both model a WuRxbased MAC protocol, and to model two other state-of-the art MAC protocols for WSNs not using WuRx. Experimental power consumption and latency measurements were conducted to validate the proposed framework and the MAC protocol leveraging WuRx. Analytical results show the convenience of using WuRx and quantify the benefits of this emerging technology. These results demonstrate that using WuRx achieves up to 135 times lower power consumption and up to 23 times lower latency compared to traditional approaches in typical low throughput WSNs applications.
\end{abstract}

\section{INTRODUCTION}

WSNs are characterized by limited resources such as memory, computation capabilities and especially available energy. Energy is typically the limiting factor of long-term applications, therefore maximizing the lifetime of WSNs is an aggressively pursued goal. Communication is usually one of the most energy consuming tasks, thus several techniques were proposed to increase the energy efficiency of communication [1]. The most popular one is duty-cycling, which consists of switching on and off the transceiver according to a predefined schedule. However, duty-cycling does not eliminate energy waste due to idle listening and incurs high latencies. Many approaches based on duty-cycling were proposed in literature and in commercial solutions [2], which typically face a hard trade-off, reducing either idle listening or latency.

Recent progress in microelectronics has made possible the design of Ultra-Low Power (ULP) WuRx which allow a continuous channel monitoring while consuming orders of magnitude less power than traditional transceivers [3]. These devices wake up the node microcontroller (MCU) or other sleeping subsystems using interrupts when a specific signal, called Wake-up Beacon (WuB), is detected. One of the main benefits of WuRx is to enable "pure" asynchronous communication that can significantly increase the energy efficiency of communications and reduce the latency [4]-[8]. Moreover, when WuRx provide computational capabilities [9], [10], they are able to process the received data autonomously in an energy efficient manner. For instance, this feature makes possible for a $\mathrm{WuB}$ to wake up only a specific node by performing address matching on the WuRx, but it also allows the WuRx to take actions without waking-up the main $\mathrm{MCU}$, e.g. activating a node sub-system or changing the sensor sampling rate, with a significant amount of energy saved.

To ensure ultra-low power consumption, state-of-the-art WuRx are usually characterized by their lower sensitivity and their lower bitrate compared with traditional WSN transceivers [3], [9], [10]. Due to this drawback, WuBs must be sent at a higher transmission power and a lower bitrate than the other frames received by the main radio to achieve the same transmission range, making the sending of WuBs energetically expensive. Therefore, MAC protocols leveraging WuRx must be carefully designed to really achieve more energy efficient communication than traditional approaches relying on dutycycling. To identify the application scenarios for which WuRx are advantageous compared to traditional approaches and to quantify the benefits brought by WuRx, an analytical model is required.

While many dedicated MAC models were proposed for the standard MAC IEEE 802.15.4 (e.g. [11]) and IEEE 802.11 (e.g. [12]), or for specific applications such as cooperative retransmission [13], only a few generic frameworks were proposed to model MAC protocols. In [14], the authors analyzed the performance of a set of MAC protocols in the context of low datarate WSNs. If the proposed traffic and radio models are generic, the latency and energy models are specific to each MAC, making this approach hard to extend to new protocols. Asudeh et al. [15] proposed a selection framework to choose the appropriate protocol that satisfies the requirements for a given context defined by a set of input parameters. Three categories of protocols (preamble sampling, common active period and scheduled) are defined and it is assumed that the protocols in the same category have similar performance characteristics. However, the new paradigm brought by WuRx cannot fit in any of these categories. Focusing on WuRx, Lont et al. [16] compared analytically the energy consumption of a WuRxbased MAC to two other traditional approaches. The authors focus on energy consumption, while in this work latency and reliability are also considered. Moreover, a separate analytical model was used for each MAC scheme, while we proposed in this work a generic model. In [17], an analytical comparison between WuRx-based and preamble sampling schemes was proposed. The authors show that WuRx enable lower latency if the transmit power can be scaled without limitation and reduce the energy consumption in the context of low traffic. The 
authors also suggested that the low sensitivity of WuRx can be compensated by the deployment of more nodes. However, the authors did not consider any real MAC approach, and the used analytical model was not designed to model specific MAC schemes. In this paper, we propose a framework combining both analytical results and experimental measurements to model accurately MAC protocols leveraging WuRx or using traditional duty-cycling approach. The proposed framework is based on Absorbing Markov Chains (AMCs) [18], and focuses on energy consumption, latency and reliability. Markov chains have already proved to be useful for modeling communications protocols, especially to study specific MAC protocols [19] and cross-layer designs [20]. Using experimental measurements, we have proven that the model provides accurate estimations in the context of low throughput applications, typical for WSNs [21].

To evaluate pure asynchronous WuRx-based approach, we used the transmitter initiated MAC protocol TI-WuR from [4], to which we added the Automatic Repeat reQuest (ARQ) error control scheme. Using the proposed analytical framework, we modeled the new version of TI-WuR. In addition, to evaluate both the proposed framework and the benefits of WuRx, two other state-of-the-art protocols have been modeled: (i) PWMAC [22], which focuses on low energy consumption and is based on the duty-cycling approach; (ii) IEEE 802.15.4, the most commonly employed standardized protocol in WSNs, which specifies two different modes, one that requires a controller node that coordinates the other nodes using beacon frames, and one without such a controller (beaconless mode). In this work, the beaconless mode was chosen, which requires the nodes to be "always awake" and uses the Carrier Sense Multiple Access with Collision Avoidance (CSMA/CA) approach to communicate. This protocol provides a benchmark value for latency. The average power consumption, latency and the reliability of these protocols were compared, analytically and experimentally. The principal contributions of this work are:

- A novel framework for modeling MAC protocols in the context of low throughput WSN applications.

- An analytical comparison of three protocols that were modeled using this new framework.

- Experimental measurements realized to set the model parameters using realistic and accurate energy consumption and latencies values, and to validate the accuracy of the framework.

- $\quad$ Experimental comparisons of the energy consumption and latency of the WuRx in low datarate scenarios using the TI-WuR protocol were conducted. The results of these experimentations, combined with those of the analytical comparisons, are used to discuss and quantify the benefits enabled by WuRx.

\section{GENERIC FRAMEWORK FOR MODELING MAC}

The proposed analytical framework for modeling the energy consumption, the latency and the reliability of MAC protocols is introduced in this section. For a given protocol, two AMCs are defined, one to model a packet reception operation and the other to model a packet transmission operation. Three typologies of states are defined to build an AMC:

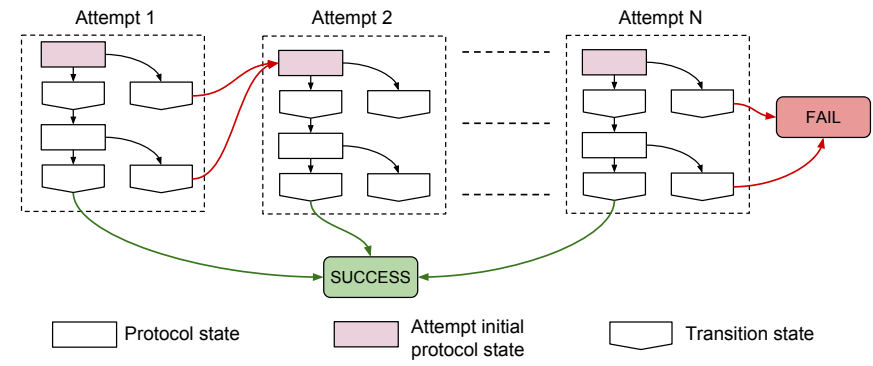

Fig. 1: Global model of an AMC modeling a packet transmission or reception with multiple attempts allowed.

protocol states, transition states and final states. Protocol states are defined by the protocol itself. To correctly evaluate the energy consumption and latency incurred by the protocol, transition states, which do not affect the behavior of the protocol, are needed to model the energy and latency cost of state transitions. Final states represent the possible outcomes of a packet transmission or reception: "Success" or "Fail", similarly to [20]. The two final states are the absorbing states of the AMCs, i.e. the states which are impossible to leave, while all the other states are transient. Fig. 1 illustrates the global structure of an AMC modeling a packet transmission or reception when multiple attempts are allowed. Each attempt has an initial state, and the initial state of the first attempt is the initial state of the AMC. On Fig. 1, transition probabilities are not shown for clarity. If an attempt fails, then a new attempt is initiated if the failed attempt was not the last one. Otherwise, the operation is a failure. In the rest of this paper, mathematical objects (AMC, matrix, vector or scalar) associated to the packet transmission operation are denoted with a " $t$ " subscript, while mathematical objects associated to the packet reception operation are denoted with a "r" subscript. When referring indifferently to both operations, the "b" subscript is used.

Let $C_{b}$ be an AMC modeling a packet transmission or reception, and $\mathbf{P}_{\mathbf{b}}$ be its associated transition matrix defined by:

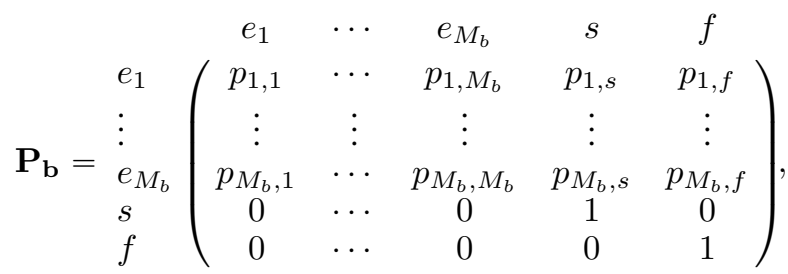

where $M_{b}$ is the number of transient states $e_{i}, i \in\left\{1, \ldots, M_{b}\right\}$, $s$ is the final "Success" state, $f$ is the final "Fail" state, and $p_{i, j} \in[0,1]$ is the transition probability from the state $e_{i}$ to the state $e_{j}$. As $s$ and $f$ are absorbing states, $p_{s, s}=p_{f, f}=1$. We denote $p_{f} \in[0,1)$ the frame failure probability, i.e., the probability that a frame transmission/reception fails. Various events can lead to a frame transmission/reception failure, such as collisions and interferences. In the proposed model, $p_{f}$ is an input parameter, allowing the evaluation of MAC protocols under various channel conditions. Hence, when constructing an AMC modeling a packet transmission or reception operation, the states corresponding to a frame transmission or reception lead to a failure with a probability $p_{f}$, and to a success with a probability $1-p_{f}$. 
Applying classical AMC results, $\mathbf{P}_{\mathbf{b}}$ can be written without loss of generality as follows [18]:

$$
\mathbf{P}_{\mathrm{b}}=\left(\begin{array}{cc}
\mathbf{Q}_{\mathrm{b}} & \mathbf{R}_{\mathbf{b}} \\
\mathbf{0} & \mathbf{I}_{\mathbf{2}}
\end{array}\right)
$$

where $\mathbf{Q}_{\mathbf{b}}$ is a $M_{b}$-by $-M_{b}$ matrix, $\mathbf{R}_{\mathbf{b}}$ is a $M_{b}$-by -2 matrix, $\mathbf{I}_{2}$ is the identity matrix of size 2 and $\mathbf{0}$ is the $2-$ by $-M_{b}$ null matrix. The fundamental matrix of $C_{b}$ is [18]:

$$
\mathbf{N}_{\mathbf{b}}=\left(\mathbf{I}_{\mathbf{M}_{\mathbf{b}}}-\mathbf{Q}_{\mathbf{b}}\right)^{-1}=\sum_{k=0}^{\infty} \mathbf{Q}_{\mathbf{b}}{ }^{k}
$$

where $\mathbf{Q}_{\mathbf{b}}{ }^{0}=\mathbf{I}_{\mathbf{M}_{\mathbf{b}}}$. The $i j$-entry of $\mathbf{N}_{\mathbf{b}}$, denoted $n_{i j}$, is the expected number of times the chain was in the state $e_{j}$ if it started in the state $e_{i}$ before being absorbed. It is assumed without loss of generality that the initial state of $C_{b}$ is $e_{i_{0}}$ with $i_{0} \in\left\{1, \ldots, M_{b}\right\}$. Thus, only the $i_{0}^{\text {th }}$ row of the $\mathbf{N}_{\mathbf{b}}$ matrix is considered, and the vector of size $M_{b}$ corresponding to this row is denoted $\mathbf{n}_{\mathbf{b}}$.

\section{A. Probability of packet operation success}

To evaluate the reliability of a protocol, the probability that a packet transmission or reception succeed, i.e. that $C_{b}$ is absorbed by the state $s$, is considered. The absorption probability matrix denoted $\mathbf{B}_{\mathbf{b}}$ is a $M_{b}-$ by -2 matrix in which the $i j-$ entry denoted $b_{i j}$ is the probability that the matrix will be absorbed by the $j^{t h}$ absorbing state if it starts in the $i^{t h}$ transient state. We have [18]:

$$
\mathbf{B}_{\mathbf{b}}=\mathbf{N}_{\mathbf{b}} \mathbf{R}_{\mathbf{b}} \text {. }
$$

As the initial state is $e_{i_{0}}$, only the $i_{0}^{\text {th }}$ row is considered and the vector of size 2 corresponding to this row is denoted $\mathbf{b}_{\mathbf{b}}$. We denote $b_{b, s}$ the entry of this vector corresponding to the probability that the chain $C_{b}$ is absorbed by the state $s$.

\section{B. Energy cost of a packet transmission/reception operation}

Let $\mathbf{e}_{\mathbf{b}}$ be the energy costs vector, i.e. the vector of size $M_{b}$ in which the $i^{t h}$ entry is the energy cost incurred by the MAC protocol when traversing the state $e_{i}$. Hence, the average energy cost of a packet transmission or reception modeled by $C_{b}$ is the scalar product of $\mathbf{n}_{\mathbf{b}}$ and $\mathbf{e}_{\mathbf{b}}$ :

$$
\overline{e_{b}}=\mathbf{n}_{\mathbf{b}} \cdot \mathbf{e}_{\mathbf{b}} .
$$

In (5), $\mathbf{n}_{\mathbf{b}}$ is related to the protocol functioning, while $\mathbf{e}_{\mathbf{b}}$ is related to application and hardware specifics, i.e. size of frames and transceiver power consumption.

\section{Average power consumption}

Knowing the expected costs of a packet transmission and a packet reception, respectively denoted $\overline{e_{t}}$ and $\overline{e_{r}}$ and computed using (5), the average power consumption incurred by communications can be computed as follows:

$$
\overline{P_{C}}=\lambda_{r} \overline{e_{r}}+\left(\lambda_{g}+b_{r, s} \lambda_{r}\right) \overline{e_{t}}+P_{o},
$$

if it is assumed that a node forwards all the packets that are successfully received. In (6), $\lambda_{r}$ and $\lambda_{g}$ are respectively the average packet reception and local packet generation rate, $b_{r, s}$ is the probability that a packet reception succeed and is computed as described in Section II-A, and $P_{o}$ is the power consumption overhead incurred by the MAC protocol and due to other activities than packet transmissions or receptions (e.g. use of WuRx, periodic transceiver switch-on...).

\section{Latency of a packet transmission operation}

We define the latency costs vector denoted $\mathrm{l}_{\mathrm{b}}$ as the vector of size $M_{b}$ in which the $i^{t h}$ entry is the latency incurred by the MAC protocol when traversing the state $e_{i}$. However, the same reasoning as in (5) can not be applied for the latency, as the scalar product of $\mathbf{n}_{b}$ and $\mathbf{l}_{\mathrm{b}}$ gives the average duration of a packet transmission or reception operation without regard to its outcome. However, when the latency is evaluated, we are interested in the packet transmission or reception duration when that succeeds. Therefore, the conditional fundamental matrix denoted $\mathbf{N}_{\mid \mathbf{a}, \mathbf{b}}$ is introduced as the matrix in which the $i j$-entry, denoted $n_{\mid a, i j}$, is the expected number of times the chain was in the state $e_{j}$ if it started in the state $e_{i}$ and knowing that the chain was absorbed by the state $a \in\{s, f\}$. Moreover, we have:

$$
n_{\mid a, i j}=\frac{b_{j a}}{b_{i a}} n_{i j} .
$$

Proof: Let $X_{i j}^{(m)}$ be the random variable that takes the value 1 if the chain is at the transient state $e_{j}$ at the step $m$ if it started at the transient state $e_{i}$, and 0 otherwise. Then:

$$
\operatorname{Pr}\left(X_{i j}^{(m)}=1\right)=q_{i j}^{(m)},
$$

where $q_{i j}^{(m)}$ is the $i j$-entry of the matrix $\mathbf{Q}_{\mathbf{b}}^{\mathbf{m}}$. Suppose $A_{i}$ is the random variable corresponding to the state which will absorb the chain if it started at the transient state $e_{i}$. According to Bayes' theorem:

$$
\begin{aligned}
\operatorname{Pr}\left(X_{i j}^{(m)}=1 \mid A_{i}=a\right) & \\
= & \frac{\operatorname{Pr}\left(A_{i}=a \mid X_{i j}^{(m)}=1\right) \operatorname{Pr}\left(X_{i j}^{(m)}=1\right)}{\operatorname{Pr}\left(A_{i}=a\right)} .
\end{aligned}
$$

Because of the Markov property:

$$
\operatorname{Pr}\left(A_{i}=a \mid X_{i j}^{(m)}=1\right)=\operatorname{Pr}\left(A_{j}=a\right),
$$

hence, we have:

$$
\begin{aligned}
\operatorname{Pr}\left(X_{i j}^{(m)}=1 \mid A_{i}=a\right)=\frac{\operatorname{Pr}\left(A_{j}=a\right)}{\operatorname{Pr}\left(A_{i}=a\right)} & \operatorname{Pr}\left(X_{i j}^{(m)}=1\right) \\
= & \frac{b_{j a}}{b_{i a}} q_{i j}^{(m)},
\end{aligned}
$$

using the definition of the absorption probabilities matrix. The expected number of times the chain was in the transient state $e_{j}$ if it started in the transient state $e_{i}$ and if it was absorbed by the state $a$ after $m$ steps is:

$$
\begin{aligned}
\mathbb{E}\left(X_{i j}^{(0)}+\cdots+X_{i j}^{(m)} \mid A_{i}\right. & =a) \\
& =\frac{b_{j a}}{b_{i a}}\left(q_{i j}^{(0)}+\cdots+q_{i j}^{(m)}\right),
\end{aligned}
$$

because of the linearity of the expectancy. Hence, when $m$ goes to infinity:

$$
\begin{aligned}
n_{\mid a, i j}= & \lim _{m \rightarrow+\infty} \mathbb{E}\left(X_{i j}^{(0)}+\cdots+X_{i j}^{(m)} \mid A_{i}=a\right) \\
& =\lim _{m \rightarrow+\infty} \frac{b_{j a}}{b_{i a}}\left(q_{i j}^{(0)}+\cdots+q_{i j}^{(m)}\right)=\frac{b_{j a}}{b_{i a}} n_{i j} .
\end{aligned}
$$

where the last equality is because of (3). 
The conditional fundamental matrix $\mathbf{N}_{\mid \mathbf{s}, \mathbf{t}}$ is such that the $i j$-entry is the expected number of times the chain was in the state $e_{j}$ if it started in the state $e_{i}$ knowing that the packet transmission succeed. As the initial state is assumed to be $e_{i_{0}}$, the vector corresponding to the $i_{0}^{t h}$ row and denoted $\mathbf{n}_{\mid \mathbf{s}, \mathbf{t}}$ is considered. Hence, the expected latency incurred by the transmission of a packet is:

$$
\bar{l}_{t}=\mathbf{n}_{\mid \mathbf{s}, \mathbf{t}} \cdot \mathbf{l}_{\mathbf{t}} \text {. }
$$

\section{E. Average number of attempts}

Let $\mathbf{c}$ be the vector of size $M_{b}$ in which the $i^{\text {th }}$ entry takes the value 1 if $e_{i}$ corresponds to the initial state of an attempt, 0 otherwise. Then, the excepted number of attempts denoted $a_{b}$, without considering the outcome of the transmission or reception operation, is given by:

$$
a_{b}=\mathbf{n}_{\mathbf{b}} \cdot \mathbf{c} .
$$

\section{MODELING THREE MAC PROTOCOLS}

In this section, three MAC protocols are modeled using the framework previously introduced to compare their power consumption, latency and reliability. The three evaluated protocols are TI-WuR, a transmitter initiated MAC leveraging WuRx extended in this paper, PW-MAC, and IEEE 802.15.4 MAC beaconless mode, which relies on CSMA/CA. For each of these protocols, both the AMC describing a packet transmission and the AMC describing a packet reception were established, and the value of $P_{o}$ was calculated. Although the same variable names are used for the three protocols $\left(P_{o}, e_{i} \ldots\right)$ for clarity, they take different values for different protocols.

\section{A. $T I-W u R$}

TI-WuR achieves a pure-asynchronous communication with WuRx. A packet transmission using the extended TIWuR is shown by Fig. 2a. The sender first sends a WuB to the WuRx of the receiving node. When it receives the $\mathrm{WuB}$, the receiving node listens to the channel using its main transceiver to receive the data frame. Afterward, the sender sends the data frame, and waits for an acknowledgment (ACK). Finally, the receiver sends the ACK when it has successfully received the data frame. Each frame sending or reception corresponds to a transient protocol state of the AMCs used to model packet transmission or reception.

In this work, the WuRx designed and developed in [9] is employed. This WuRx works in the $868 \mathrm{MHz}$ frequency bands, and receives data with On-Off Keying modulation (OOK) at a bitrate of $1 \mathrm{kbps}$. The sensitivity in these conditions was measured to be $-55 \mathrm{dBm}$, with a range around $25 \mathrm{~m}$ using $3 \mathrm{dBi}$ gain antennas. The WuRx provides computational capabilities due to the ULP MCU, the PIC12LF1552 from Microchip, that it embeds. The power consumption of the whole WuRx was measured to be $1.83 \mu \mathrm{W}$ in always-on listening mode and $284 \mu \mathrm{W}$ when receiving and processing data with the ULP MCU active. The ULP MCU was programmed to partially incorporate TI-WuR, and in particular to perform address matching, allowing nodes to wake up only a specific node and not all their neighbors.

Due to the presence of the WuRx, the main transceiver is only used for sending and receiving data, and therefore

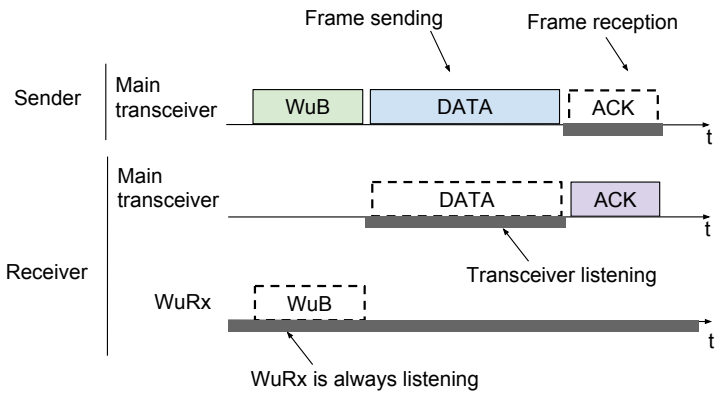

(a) Packet transmission using TI-WuR.

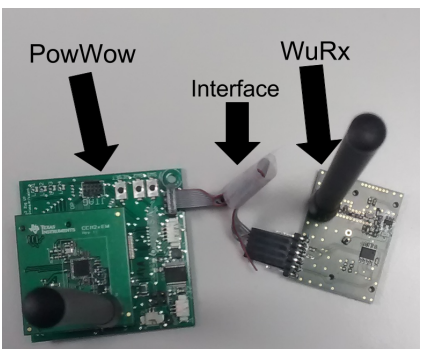

(b) A PowWow node equipped with a WuRx.

Fig. 2: Packet transmission using TI-WuR and platform setup.

the energy consumption it incurs is accounted by the two first terms of (6). However, $P_{o}$ must account for the power consumption due to the always on WuRx, although it is very low. At each WuB reception, the WuRx ULP MCU wakes up to process the data embedded in the $\mathrm{WuB}$. As this is part of the packet reception operation, it is accounted in the first term of (6). The expected number of attempts per packet reception $a_{r}$ corresponds to the expected number of $\mathrm{WuB}$ received per packet reception when accounting retransmissions. Hence, we have:

$$
P_{o}=\left(1-a_{r} \lambda_{r} \frac{L_{w}}{R_{w}}\right) P_{w}^{l},
$$

where $L_{w}$ is the size of a $\mathrm{WuB}, R_{w}$ is the bitrate at which the WuBs are transmitted and $P_{w}^{l}$ is the power consumption of the WuRx when only the analog front-end is active for listening to the medium.

\section{B. PW-MAC}

PW-MAC is a receiver initiated protocol based on the traditional duty-cycling approach that focuses on low energy consumption for both the sender and the receiver [22]. Each node periodically sends a beacon to inform the neighbors that it is ready to receive, and then listen to the channel. If no data frame is detected after a short delay, the node goes back to the sleep mode. Each node "learns" the wake-up schedule of its forwarders, and if it has a packet to send to one of them, it wakes up just before the next scheduled wake-up of the forwarder to receive the periodic beacon that initiates the communication. This approach significantly reduces the idle listening on the transmitting node side, but not on the received node side as periodic beacon sending and channel listening are still required. 
During a packet reception, the beacon transmission is considered as being part of the packet reception operation. The expected number of attempts per packet reception operation $a_{r}$ corresponds to the expected number of beacons transmitted per packet reception operation when counting retransmissions. Hence, the power consumption overhead incurred by the periodic switch-on of the transceiver is:

$$
P_{o}=\left(\frac{1}{T_{W I}}-a_{r} \lambda_{r}\right) e_{w}
$$

where $e_{w}$ is the energy cost of a scheduled wake-up, and $T_{W I}$ is the wake-up interval, i.e. the average duration between two scheduled wake-ups.

\section{Unslotted CSMA/CA}

Unslotted CSMA/CA, which is used by IEEE 802.15.4 MAC layer beaconless mode, requires the transceiver to be always listening to the channel. As the cost of packets transmissions and receptions are accounted by the two first terms of (6), the power overhead incurred by CSMA/CA is:

$$
P_{o}=\left(1-\lambda_{r}\left(\mathbf{n}_{\mathbf{r}} \cdot \mathbf{l}_{\mathbf{r}}\right)-\left(\lambda_{g}+b_{r, s} \lambda_{r}\right)\left(\mathbf{n}_{\mathbf{t}} \cdot \mathbf{l}_{\mathbf{t}}\right)\right) P_{r x},
$$

where $P_{r x}$ is the power consumption of the transceiver when it is listening the channel. In (18), $\left(\mathbf{n}_{\mathbf{r}} \cdot \mathbf{l}_{\mathbf{r}}\right)$ and $\left(\mathbf{n}_{\mathbf{t}} \cdot \mathbf{l}_{\mathbf{t}}\right)$ correspond to the expected duration of a packet reception and packet transmission operation respectively, without considering operation outcomes.

\section{ANALYTICAL AND EXPERIMENTAL RESULTS}

In this section, analytical results and experimental measurements are presented to compare the power consumption, reliability and latency of TI-WuR, PW-MAC and CSMA/CA. Microbenchmarks of these protocols were conducted to obtain accurate values of the energy consumption and latency incurred by each state of these protocols. These values were used to set the energy costs and latency costs vectors, as detailed in Section IV-A. Section IV-B exposes analytical results along with results of experimental measurements realized to evaluate the accuracy of the proposed models. All the experimentations were realized using the PowWow platform [23] and the WuRx from [9]. However, the WuRx were connected to the nodes only when evaluating TI-WuR, and the obtained setup is shown by Fig. $2 b$.

\section{A. Microbenchmarks}

The proposed analytical model requires the energy cost vectors $\mathbf{e}_{\boldsymbol{t}}$ and $\mathbf{e}_{\mathbf{r}}$ and the latency cost vectors $\mathbf{l}_{\mathbf{t}}$ and $\mathbf{l}_{\mathbf{r}}$ to be carefully set to provide realistic power consumption and latency values. Fig. 3 shows the traces obtained for the extended TI-WuR, which were acquired using a Keysight N6705B DC power analyzer. Fig. 3a exposes a packet transmission operation, which includes among others the sending of the WuB (C), the sending of the data frame (D) and the reception of the ACK $(\mathrm{E})$. The sending of the WuB is preceded by a radio setup phase (B) and a $\mathrm{MCU}$ processing phase (A). Moreover, MCU processing stages are also present between frames receptions/transmissions. Fig. $3 b$ shows in detail the different stages of a packet reception using TIWuR. The processing of the WuB by the WuRx can be

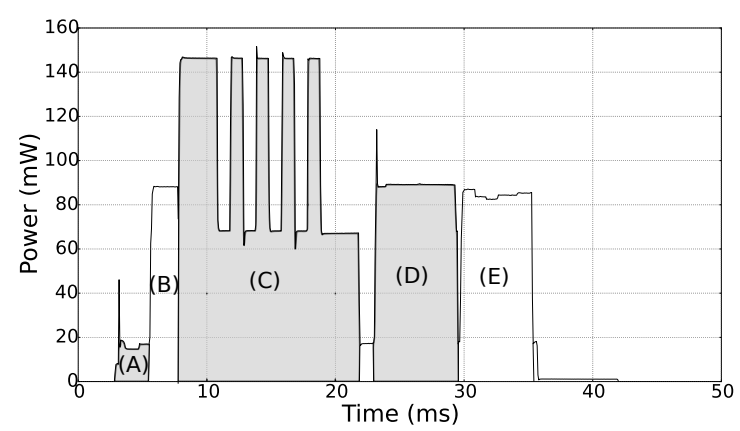

(a) Sending a packet using TI-WuR.

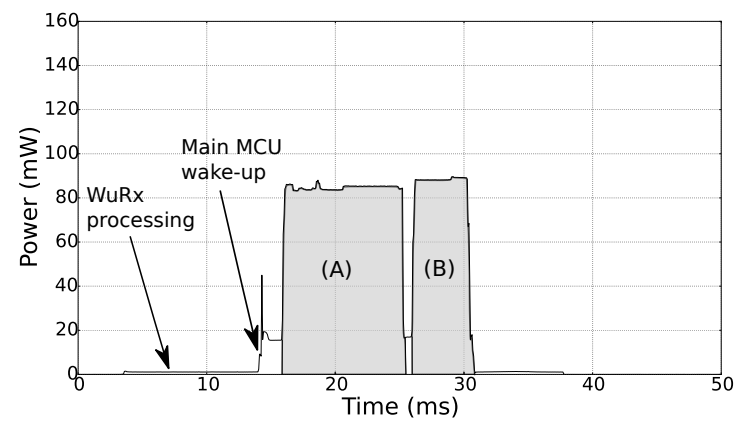

(b) Receiving a packet using TI-WuR.

Fig. 3: Microbenchmarks of TI-WuR.

seen, as well as the data frame reception (A) and the ACK transmission (B). Moreover, similarly to what was observed for the packet transmission, phases of MCU processing are present juster after the main MCU wake-up and between frames transmission/reception. These measurements were used to set the energy costs and latency costs vectors needed by the TI-WuR model. Similarly, microbenchmarks were conducted for PW-MAC and CSMA/CA, and the energy costs and latency costs vectors associated to these protocols were set using the same procedure.

Moreover, when transmitting a packet in PW-MAC, a node has to wait in average $\frac{T_{W I}}{2} \mathrm{~S}$ for the receiving node to wake up, and, if the attempt fails, $T_{W I}$ s for the next attempt. In TI-WuR or CSMA/CA, the node can start the transmission immediately. However, if the attempt fails, a backoff is set before starting a new attempt. These latency costs were accounted in the $\mathbf{l}_{\mathbf{t}}$ and $l_{r}$ vectors, and therefore are considered by (14).

\section{B. Results and Discussion}

Analytical results are presented and discussed in this section, along with experimental measurements that were carried out to evaluate the accuracy of the proposed model. The average power consumption and the packet transmission latency of a testbed node that locally generates packets at a rate $\lambda_{g}$ and receives packets at a rate $\lambda_{r}$ were measured. The node forwards all the packets that it successfully receives. Measurements were realized for various values of $\lambda_{g}, \lambda_{r}$, the wake-up interval $T_{W I}$ when PW-MAC was evaluated, and the WuB size $L_{w}$ when TIWuR was evaluated. The analytical and experimental results are presented by Fig. 4, on which the red markers represent the experimentally measured values. It can be observed that the 


\begin{tabular}{|c|c|c|c|c|c|}
\hline $\begin{array}{l}\text { TI-WuR, } L_{w}=1 \mathrm{~B} \quad R_{w}=1 \mathrm{kbps} \text { Analytical } \\
\text { TI-WuR, } L_{w}=2 \mathrm{~B} \quad R_{w}=1 \mathrm{kbps} \text { Analytical } \\
\text { TI-WuR, } L_{w}=4 \mathrm{~B} R_{w}=1 \mathrm{kbps} \text { Analytical }\end{array}$ & 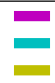 & $\begin{array}{l}\text { TI-WuR, } L_{w}=8 \mathrm{~B} \quad R_{w}=1 \mathrm{kbps} \text { Analytical } \\
\text { TI-WuR, } L_{w}=8 \mathrm{~B} R_{w}=10 \mathrm{kbps} \text { Analytical } \\
\text { TI-WuR, } L_{w}=8 \mathrm{~B} \quad R_{w}=20 \mathrm{kbps} \text { Analytical }\end{array}$ & 11 & $\begin{array}{l}\text { PW-MAC Analytical } \\
\text { CSMA/CA Analytical } \\
\text { TI-WuR Experimental }\end{array}$ & $\begin{array}{l}\star \star \text { PW-MAC Experimental } \\
\Delta \Delta \text { CSMA/CA Experimental }\end{array}$ \\
\hline
\end{tabular}

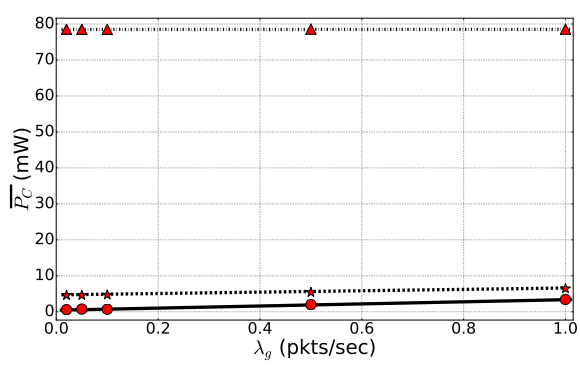

(a) $\overline{P_{c}}$ as a function of $\lambda_{g}$.

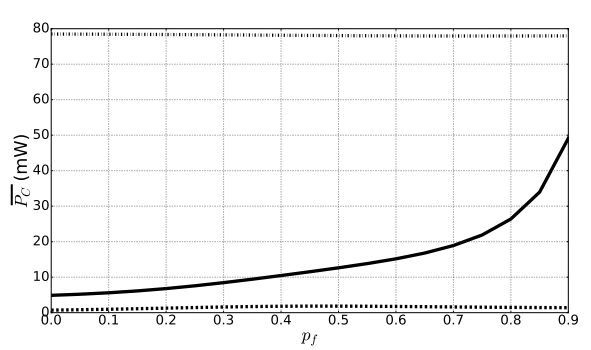

(d) $\overline{P_{c}}$ as a function of $p_{f}$.

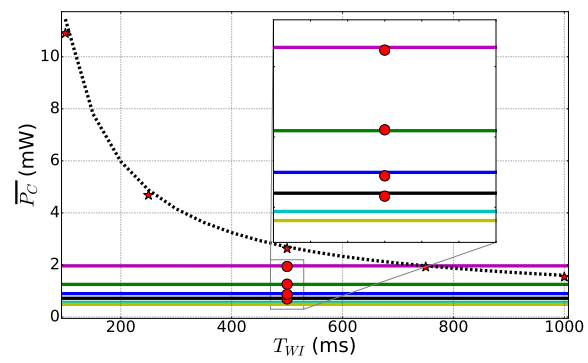

(b) $\overline{P_{c}}$ as a function of $T_{W I}, L_{w}$ and $R_{w}$.

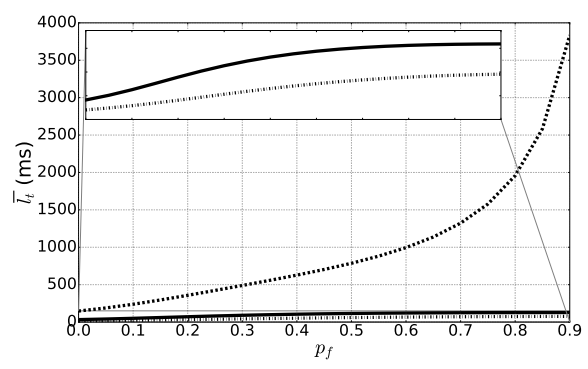

(e) $\overline{l_{t}}$ as a function of $p_{f}$.

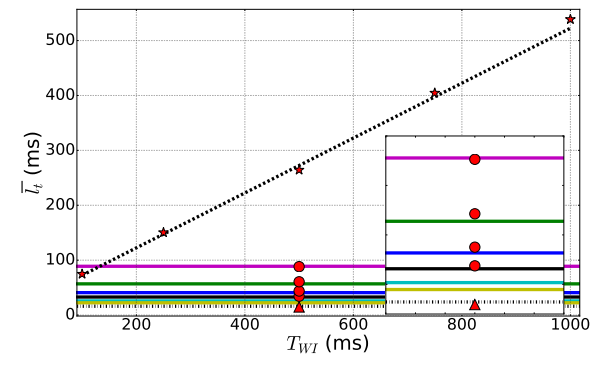

(c) $\overline{l_{t}}$ as a function of $T_{W I}, L_{w}$ and $R_{w}$.

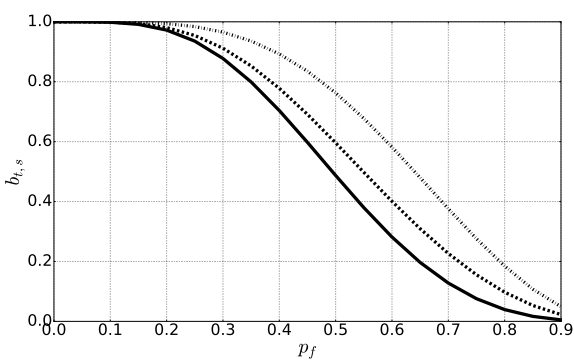

(f) $b_{t, s}$ as a function of $p_{f}$.

Fig. 4: Analytical and experimental results.

analytical models fit the experimental measurements well for the three protocols, therefore these results show the accuracy of the proposed framework.

Fig. 4a shows the impact of $\lambda_{g}$ on the average consumed power $\overline{P_{c}}$ when $T_{W I}=250 \mathrm{~ms}, R_{w}=1 \mathrm{kbps}$ and $L_{w}=1$ byte. As expected from (6), $\overline{P_{c}}$ increases linearly with $\lambda_{g}$ and $\lambda_{r}$, and therefore similar results were obtained for $\lambda_{r}$, which are not shown in this paper. The average power consumption of the node with TI-WuR is at least twice lower than with PW-MAC, which can be explained by the fact that TI-WuR does not require periodic switch-on of the transceiver. As CSMA/CA requires the transceiver to be constantly listening to the channel, it consumes between 135 and 23 times more than TI-WuR and between 17 and 12 times more than PWMAC depending of $\lambda_{g}$. These results show the energy saving enabled by WuRx in low throughput applications.

Fig. 4b exposes $\overline{P_{c}}$ when $T_{W I}, L_{w}$ and $R_{w}$ vary. The power consumption of CSMA/CA is not shown, as it is up to 135 times higher than for the two other evaluated protocols. Higher values of $T_{W I}$ leads to lower power consumption of PW-MAC, as the duty-cycle of the transceiver decreases with $T_{W I}$. For values of $T_{W I}$ higher than $750 \mathrm{~ms}$, the power consumption of PW-MAC becomes lower that the one of TI-WuR when $L_{w}=8$ bytes and $R_{w}=1 \mathrm{kbps}$. However, this is at the cost of higher latency as shown by Fig. 4c. These results show the convenience of using WuRx in low throughput applications, as in the case of many WSNs scenarios, when low latency and low power consumption is required. Indeed, as PW-MAC relies on the duty-cycling approach, a node has to wait for its forwarder to turn its transceiver on before being able to engage a communication with it, and the average waiting time is $\frac{T_{W I}}{2}$. Therefore, a compromise between power consumption and latency must be made using the traditional duty-cycling approach. When high bitrates are used for sending the WuB (10 kbps or $20 \mathrm{kbps}$ ), TI-WuR significantly outperforms PWMAC regarding both the power consumption and the latency, even when the WuB size is 8 bytes as it can be seen on Fig. $4 \mathrm{~b}$ and Fig. 4c. Because the WuRx from [9] is optimized for a bitrate of $1 \mathrm{kbps}$, using higher bitrate was not realized experimentally. The Fig. 4c also shows that the latency incurred by CSMA/CA is lower than both TI-WuR and PW-MAC, as it does not require any beacon sending or rendez-vous scheme. However, this is at the cost of letting the main transceiver always listening, incurring a power consumption of one order of magnitude higher than with TI-WuR. Moreover, using TIWuR with $R_{w}=10 \mathrm{kbps}$ permits to achieve a latency of $25.9 \mathrm{~ms}\left(22.0 \mathrm{~ms}\right.$ if $\left.R_{w}=20 \mathrm{kbps}\right)$, while the latency of CSMA/CA is $16.2 \mathrm{~ms}$ in the same conditions.

Fig. 4d shows the impact of the frame failure probability $p_{f}$ on $\overline{P_{c}}$ when $T_{W I}=250 \mathrm{~ms}, R_{w}=1 \mathrm{kbps}$ and $L_{w}=1$ byte. It can be seen that while $p_{f}$ has a low impact on the average power consumption incurred by TI-WuR and PW-MAC, it has a strong impact on the average power consumption incurred by PW-MAC. Indeed, when a node sends a packet in PWMAC to a forwarder node, the sending node first wakes up just before the forwarder sends its scheduled beacon. If no beacon is received, then the sending node assumes that the synchronization with the forwarder node was lost, keeping its transceiver active until a beacon from the forwarder node is received to resynchronize. Therefore, a high frame failure 
probability (e.g. due to collisions or interferences) causes frequent resynchronization activities and therefore significantly increases the power consumption. This unwanted effect also leads to high packet transmission latency when $p_{f}$ becomes high as shown by Fig. 4e, as the sending node waits for a valid beacon from the forwarder node. On the other hand, when an attempt to transmit a packet fails with TI-WuR of CSMA/CA, the sending node makes a new attempt until the transmission succeed or exceed a predefined number of re-transmission (set to 4 in this work), each re-transmission being preceded by a random backoff. Nonetheless, the functioning of PWMAC leads to higher probability of successfully delivering the packet compared to TI-WuR as shown by Fig. 4f. However, the difference becomes significant when the probability of frame failure is higher than $20 \%$. CSMA/CA is the more reliable protocol, as only two frames must successfully be transmitted for the communication to be successful (the data frame and the ACK), while three frames must be successfully transmitted for TI-WuR and PW-MAC.

\section{CONCLUSION}

In this paper, a new framework to model MAC protocols that focuses on power consumption, latency and reliability was introduced. This framework has been employed to model PW-MAC, IEEE 802.15.4 beaconless mode, and TIWuR, a protocol leveraging wake-up extended in this work. Experimental measurements demonstrated the validity of the model. Moreover, experimental and analytical results shown that WuRx enables significant power saving compared to PWMAC, while incurring a latency close to the one of CSMA/CA. However, CSMA/CA requires the transceiver to be always on to listen the medium. Moreover, as for CSMA/CA, the latency and power consumption of TI-WuR are not strongly impacted by the frame failure probability. On the contrary, PW-MAC incurs high power consumption and high latency in bad channel condition. These results shown that using WuRx in low throughput WSNs applications allows both low power consumption and low latency, while using traditional approaches based on duty-cycling requires a compromise between these two performance metrics.

\section{REFERENCES}

[1] T. Rault, A. Bouabdallah, and Y. Challal, "Energy efficiency in wireless sensor networks: A top-down survey," Computer Networks, vol. 67, pp. $104-122,2014$

[2] P. Huang, L. Xiao, S. Soltani, M. Mutka, and N. Xi, "The Evolution of MAC Protocols in Wireless Sensor Networks: A Survey," IEEE Communications Surveys Tutorials, vol. 15, no. 1, pp. 101-120, January 2013.

[3] N. S. Mazloum and O. Edfors, "Performance Analysis and Energy Optimization of Wake-Up Receiver Schemes for Wireless Low-Power Applications," IEEE Transactions on Wireless Communications, vol. 13, no. 12, pp. 7050-7061, December 2014.

[4] J. Oller, I. Demirkol, J. Casademont, J. Paradells, G. Gamm, and L. Reindl, "Has Time Come to Switch From Duty-Cycled MAC Protocols to Wake-Up Radio for Wireless Sensor Networks ?" IEEE/ACM Transactions on Networking, 2015.

[5] F. Sutton, B. Buchli, J. Beutel, and L. Thiele, "Zippy: On-Demand Network Flooding," in Proceedings of the 13th ACM Conference on Embedded Networked Sensor Systems (SenSys), November 2015.

[6] D. Spenza, M. Magno, S. Basagni, L. Benini, M. Paoli, and C. Petrioli, "Beyond duty cycling: Wake-up radio with selective awakenings for long-lived wireless sensing systems," in IEEE Conference on Computer Communications (INFOCOM), April 2015.
[7] H. Yomo, K. Abe, Y. Ezure, T. Ito, A. Hasegawa, and T. Ikenaga, "Radio-On-Demand Sensor and Actuator Networks (ROD-SAN): System Design and Field Trial," in IEEE Global Communications Conference (GLOBECOM), December 2015.

[8] V. raja Karuppiah Ramachandran, B. J. van der Zwaag, N. Meratnia, and P. J. Havinga, "Evaluation of MAC Protocols with Wake-up Radio for Implantable Body Sensor Networks," in Fourth International Conference on Selected Topics in Mobile \& Wireless Networking (MoWNet), 2014.

[9] M. Magno and L. Benini, "An ultra low power high sensitivity wake-up radio receiver with addressing capability," in IEEE 10th International Conference on Wireless and Mobile Computing, Networking and Communications (WiMob), October 2014.

[10] Y. Ammar, S. Bdiri, and F. Derbel, "An ultra-low power wake up receiver with flip flops based address decoder," in 12th International Multi-Conference on Systems, Signals Devices (SSD), March 2015.

[11] X. Vilajosana, Q. Wang, F. Chraim, T. Watteyne, T. Chang, and K. S. J. Pister, "A Realistic Energy Consumption Model for TSCH Networks," IEEE Sensors Journal, vol. 14, no. 2, pp. 482-489, February 2014.

[12] C.-E. Weng and H.-C. Chen, "The performance evaluation of IEEE 802.11 DCF using Markov chain model for wireless LANs," Computer Standards \& Interfaces, vol. 44, pp. 144 - 149, 2016.

[13] B. Hagelstein, M. Abolhasan, D. Franklin, and F. Safaei, "A general performance model for MAC layer cooperative retransmission contention protocols," in IEEE Global Communications Conference (GLOBECOM), December 2013, pp. 1584-1589.

[14] K. Langendoen and A. Meier, "Analyzing MAC Protocols for Low Data-Rate Applications," in ACM Transactions on Sensor Networks (ToSN), vol. 7, no. 1,2010 , pp. 1-34.

[15] A. Asudeh, G. V. Záruba, and S. K. Das, "A general model for MAC protocol selection in wireless sensor networks," Ad Hoc Networks, vol. 36, Part 1, pp. 189 - 202, 2016.

[16] M. Lont, D. Milosevic, P. G. M. Baltus, A. H. M. van Roermund, and G. Dolmans, "Analytical Models for the Wake-Up Receiver Power Budget for Wireless Sensor Networks," in IEEE Global Telecommunications Conference (GLOBECOM), November 2009.

[17] R. Su, T. Watteyne, and K. Pister, "Comparison between Preamble Sampling and Wake-Up Receivers in Wireless Sensor Networks," in Global Telecommunications Conference (GLOBECOM 2010), 2010 IEEE, December 2010.

[18] C. M. Grinstead and J. L. Snell, Grinstead and Snell's Introduction to Probability. Chance Project, 2006.

[19] Q. Zhao and L. Tong, "A dynamic queue protocol for multiaccess wireless networks with multipacket reception," IEEE Transactions on Wireless Communications, vol. 3, no. 6, pp. 2221-2231, November 2004.

[20] Y. Wang, M. Vuran, and S. Goddard, "Cross-Layer Analysis of the Endto-End Delay Distribution in Wireless Sensor Networks," IEEE/ACM Transactions on Networking, vol. 20, no. 1, pp. 305-318, February 2012.

[21] Y. Liu, Y. He, M. Li, J. Wang, K. Liu, and X. Li, "Does Wireless Sensor Network Scale? A Measurement Study on GreenOrbs," IEEE Transactions on Parallel and Distributed Systems, vol. 24, no. 10, pp. 1983-1993, October 2013.

[22] L. Tang, Y. Sun, O. Gurewitz, and D. Johnson, "PW-MAC: An energy-efficient predictive-wakeup MAC protocol for wireless sensor networks," in Proceedings of IEEE INFOCOM, April 2011.

[23] "PowWow: Power Optimized Hardware and Software FrameWork for Wireless Motes," http://powwow.gforge.inria.fr/, 2014, [Online; accessed December-2015]. 